\title{
Study on the Effect of Multi-Type Current Transformers Hybrid Operation on Differential Protection of the Bus Based on Dynamic Simulation
}

\author{
Wenbiao Liao, Zexin Zhou, Rongrong Zhan, Yanjun Li, Zhengguang Chen \\ CEPRI, Beijing, China \\ Email: 1048593969@qq.com
}

How to cite this paper: Liao, W.B., Zhou, Z.X., Zhan, R.R., Li, Y.J. and Chen, Z.G. (2017) Study on the Effect of Multi-Type Current Transformers Hybrid Operation on Differential Protection of the Bus Based on Dynamic Simulation. Energy and Power Engineering, 9, 1-11.

https://doi.org/10.4236/epe.2017.94B001

Received: October 31, 2016

Accepted: March 30, 2017

Published: April 6, 2017

\begin{abstract}
This paper analyzes characteristics of multi type current transformers hybrid operation for each branch of the bus and their effects on differential protection of the bus. By theoretically analyzing transmission characteristics of multi type current transformers and their influence factors, we study the dynamic model testing method of multi type current transformers for the bus, and design 3 kinds of testing schemes by making the equivalent model based on the field of P-level current transformer, TPY-level current transformer and electronic current transformer, and build the hybrid operation testing platform of multi type current transformers. Finally, we compare and analyze the transmission characteristics difference of multi type current transformers on the same branch and the characteristics difference of hybrid operation in two successive external faults, analyze the cause behind the differences, and put forward the corresponding improvement measures.
\end{abstract}

\section{Keywords}

Differential Protection of the Bus, Multi-Type Current Transformer

Hybrid Operation, Dynamic Simulation Test Method, Dynamic

Simulation Test Platform

\section{Introduction}

As China's intelligent substation is entering a new era of comprehensive construction, electronic current transformer as an important digital substation basic equipment, has an essentially different working principle from electromagnetic current transformer of the conventional substation. The electronic current trans- 
former has an unparalleled advantage in the transient response, which can reflect the actual situation of Primary system more accurately. With the increase of the electronic current transformer applications, and in order to reduce the engineering quantity and reduce the cost in the process of digitalization of the conventional station, the electromagnetic current transformer and the electronic current transformer are used together in power system. For example, one branch of the bus uses P-level current transformer or electronic current transformer, the other branch uses TPY-level current transformer.

Due to the transmission characteristics difference between different types of current transformers, the use of different characteristics current transformer at different intervals may lead to protecting device malfunction. The research on the physical simulation testing system adapting to the multi-type transformer hybrid operation is still empty. Therefore, based on the analysis of the difference of transmission characteristics for protective current transformer, this paper proposes electromagnetic current transformer models (including P-level and TPY-level) and the electronic current transformer models that are equivalent to the actual current transformers. The proposed model takes bus protection as an example, builds the testing platform of multi-type current transformer hybrid operation according to the operation of practical substation, which can be used to study the operating characteristics of multi-type current transformer hybrid operation, and the effects on the action of bus differential protection.

\section{Analysis of Multi-Type Current Transformer Transmission Characteristics}

\subsection{Analysis of Electromagnetic Type Current Transformer Transmission Characteristics}

\subsubsection{Transient Transmission Characteristics of Electromagnetic Type Current Transformer}

When an asymmetrical fault occurs in primary system, its transient short-circuit current expression [1] is shown as follows:

$$
i_{p}(t)=\sqrt{2} I_{p s c}\left[e^{\frac{t}{T_{p}}} \cos \theta-\cos (\omega t+\theta)\right]
$$

Of which $i_{p}$ is the instantaneous value of primary current, $I_{p s c}$ is steady-state valid value of the short-circuit current, $T_{p}$ is primary system time constant, $\theta$ is offset angle of the short-circuit fault voltage, $\theta=0^{\circ}$ is the current full offset.

When the current transformer is not saturated, and iron core loss is not considered, based on the characteristics of linear excitation transmission, the solution of the expression of excitation current for the current transformer [2]:

$i_{0}(t)=\frac{\sqrt{2} I_{p s c}}{N}\left[\frac{T_{p}}{T_{p}-T_{s}} e^{-\frac{t}{T_{p}}} \cos \theta-\frac{T_{p}}{T_{p}-T_{s}} e^{-\frac{t}{T_{s}}} \cos \theta+\frac{\sin \theta}{\omega T_{s}} e^{-\frac{t}{T_{s}}}-\frac{\sin (\omega t+\theta)}{\omega T_{s}}\right]$

Of which $i_{0}$ is the excitation current, $T_{s}$ is the second system time constant. As can be seen from Equation (2), transient transmission error of the current trans- 
former is caused mainly by four parts: the rightmost is sinusoidal periodic forced components of the current transformer, the equivalent of steady-state shortcircuit current; the second item on the right is non-periodic free component of the current transformer, which compensates for the difference between the initial periodic component of the short circuit and the initial excitation current; The first term on the left is the forced non-periodic component of the current transformer excitation current, which is attenuated by the primary current time constant $T_{p}$; The second term on the left is the non-periodic free component, which compensates for the initial value of the non-periodic component at the beginning of the short-circuit. For the periodic component, the error is inversely proportional to the secondary time constant, the larger that secondary time constant, the smaller the error caused by the transmission periodic component. For non-periodic components, the excitation current has a non-periodic component attenuated by primary time constant, and it is also related to the CT secondary time constant of the current transformer.

\subsubsection{Transient Saturation Characteristics of Electromagnetic Current Transformer}

When the magnetic flux density is greater than the iron core saturation flux density $\Phi_{\text {sat }}$ the current transformer saturates and cannot be linear transmission, and the waveform distortion of the secondary current occurs. When saturation is drawing near, the proportion of the power frequency periodic component to the total magnetic flux density is very small, equivalent to adding a superimposed the power frequency periodic magnetic flux density with little amplitude value to the linearly-increasing magnetic flux density, as shown in Figure 1.

\subsection{Analysis of Electronic Current Transformer Transmission Characteristics}

Electronic current transformer has the characteristics of high precision, high

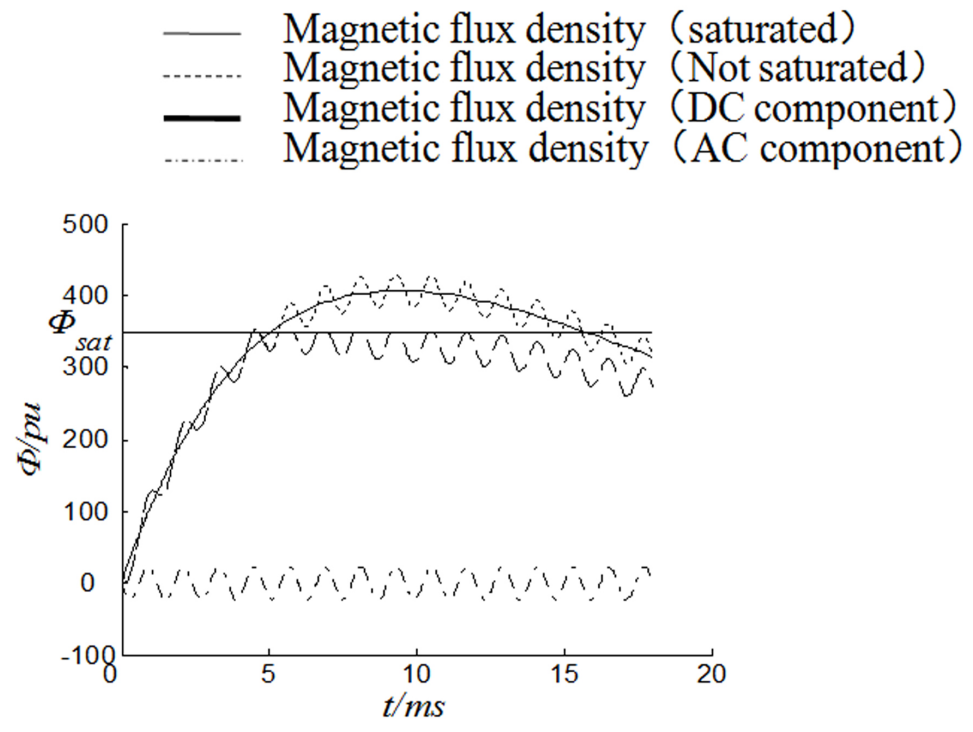

Figure 1. Magnetic flux density of current transformer transient saturation. 
reliability, wide band, non-magnetic saturation problem, anti-interference ability and so on [3] [4] [5]. It first converts the measured current signal into voltage signal by using the air-core coil sensing unit, and then converts the voltage signal into optical pulse signal through amplifier, Integrator, A/D conversion, digital signal processing and photoelectric conversion, then transmits optical pulse signal to data processing system on the low voltage side through optical fiber, and finally outputs the digital signal. Electronic current transformer equivalent circuit is shown in Figure 2. Of which, $R_{s}$ is the total resistance of the coil winding and lead; $L$ is the coil inductance; $C_{0}$ is equivalent stray capacitance of the coil; $R_{a}$ is the load resistance.

In the steady-state case, ignoring the stray capacitance $C_{0}$, the phase relationship of voltage $u(t)$ and $u_{0}(t)$ is as follows:

$$
\dot{V}_{0}\left(1+\frac{R_{s}}{R_{a}}+\frac{j \omega L}{R_{a}}\right)=\dot{U}
$$

The phase error $\varphi$ :

$$
\varphi=\operatorname{arctg}\left(\frac{\omega L}{R_{s}+R_{a}}\right)
$$

Amplitude relative error $\varepsilon$ :

$$
\varepsilon=\left|\frac{\dot{U}-\dot{V}_{0}}{\dot{U}}\right|=\left|\frac{R_{s}+j \omega L}{R_{a}+R_{s}+j \omega L}\right|=\frac{\sqrt{R_{s}^{2}+(\omega L)^{2}}}{\sqrt{\left(R_{a}+R_{s}\right)^{2}+(\omega L)^{2}}}
$$

It can be seen from the above two formulas, electronic current transformer phase error and amplitude relative errors are closely related with the coil's own parameters. In the actual calculation of the case of power frequency, $\omega L$ can be ignored, the phase error is almost zero, and the amplitude relative error is constant.

\subsection{Impact on Protection under Multi Types Current Transformer Hybrid Operation}

As the transmission characteristic of different current transformers is diverse, the use of different current transformers at different intervals may lead to protection device malfunction. 1) if all branches of the bus are using TPY-level current transformer, the transmission characteristics are basically the same, so pro-

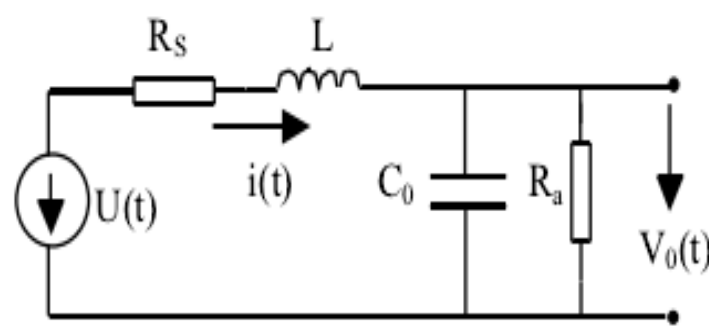

Figure 2. Equivalent circuit diagram of Electronic current transformer. 
tection device can accurately act when the fault occurs internally and externally. 2) if a branch of the bus is using P-level current transformer, the other branches are using TPY-level current transformer, the protection device can accurately act internally but not externally. for example when two consecutive faults occur externally, P-level current transformer may be seriously saturated, the existing differential protection device based on $5 \mathrm{~ms}$ saturation logic criteria may not determine Correctly and block the differential protection, therefore differential protection has the risk of malfunction. 3) if a branch of the bus is using the electronic current transformer, the other branches are using TPY-level current transformer, protection device can act accurately when the fault occurs internal and external.

\section{Dynamic Model Test System of Multi-Type Current Transformer Hybrid Operation}

\subsection{Test Scheme of Multi-Type Current Transformer Hybrid Operation}

To study the influence of using multi-type current transformers on the bus-bar protection, the multi-type current transformers includes the P-level current transformer model, the TPY-level current transformer model and the electronic current transformer model. Of which, P-level current transformer's accuracy grade value of is $5 \mathrm{P}$, limit coefficient is 20 , static saturation multiple is 5 , primary current error under less than 20 times of its rated current is less than 5 percentage. TPY-level current transformer's rated symmetrical short-circuit current factor is 20, with small air gap iron core, maximum instantaneous error under rated secondary symmetrical short-circuit current is less than peak 7.5 percentage, working cycle is $100 \mathrm{~ms}-500 \mathrm{~ms}-100 \mathrm{~ms}$, and TPY-level current transformer can simulate field current transformer with current ratio 630/5, 1250/1, $2000 / 1,3000 / 1$. The electronic current transformer is 5TPE-level, with sampling amplitude error less than 1 percentage and sampling angle error less than $60 \mathrm{~min}$ at the rated primary current, and compound error 5 percentage under the rated accuracy limits primary current.

Generally, the current transformer is configured according to the interval or voltage level. Hybrid operation of P-level current transformer and TPY-level current transformer is used to study the bus differential protection, with one specified interval using P-level current transformer, and other intervals using TPY-level current transformer. Hybrid operation of electronic current transformer and TPY-level current transformer is used to study differential protection of the bus, with the specified interval using electronic current transformer, and other intervals using TPY-level current transformer. In order to show the effect of current transformer hybrid operation of different principle on the bus differential protection, three test schemes are designed: A, B, C. of which in A scheme, all the branches of the bus are using TPY-level current transformer. In B scheme, a branch of the bus is using P-level current transformer and other branches are using TPY-level current transformer. In C scheme, a branch of the 
bus is using electronic current transformer and other branches are using TPYlevel current transformer.

\subsection{Dynamic Model Testing System}

As a mature and important technical means in the field of power system research, the physical dynamic model test has the characteristics of strong empirical, mature technology, accuracy and reliable simulation results [6] [7]. Dynamic test system is shown in Figure 3. M station and $\mathrm{N}$ station system are connected by the $500 \mathrm{kV}$ transmission lines, $\mathrm{M}$ station installs two generating units $(10 \mathrm{G}$, $12 \mathrm{G}$ ) with total capacity $2100 \mathrm{MW}$. M station is also connected to three-winding transformer, whose medium voltage side is connected to an area equivalent system and low-voltage side is connected to two $10 \mathrm{kV}$ bus branches; $\mathrm{N}$ station adopts half-switch wiring, and is connected to an area equivalent system. Length of the transmission line is $400 \mathrm{~km}$, whose positive sequence parameters are $x_{1}=$ $0.28 \Omega / \mathrm{km}, C_{1}=0.0135 \mu \mathrm{F} / \mathrm{km}, \varphi_{1}=86^{\circ}$, zero sequence parameters are $x_{0}=0.85$ $\Omega / \mathrm{km}, c_{0}=0.0093 \mu \mathrm{F} / \mathrm{km}, \varphi_{0}=76^{\circ}$, and both ends of the transmission line is equipped with shunt reactor with capacity 150 MVar.

\section{Test Results Analysis}

Based on the dynamic test system, this paper illustrates the effect of the multi-type current transformer hybrid operation on differential current, braking current and action trajectory of differential protection, taking bus differential protection as an example. Bus differential protection uses braking characteristics of two fold line ratio, the action equation is as follows:

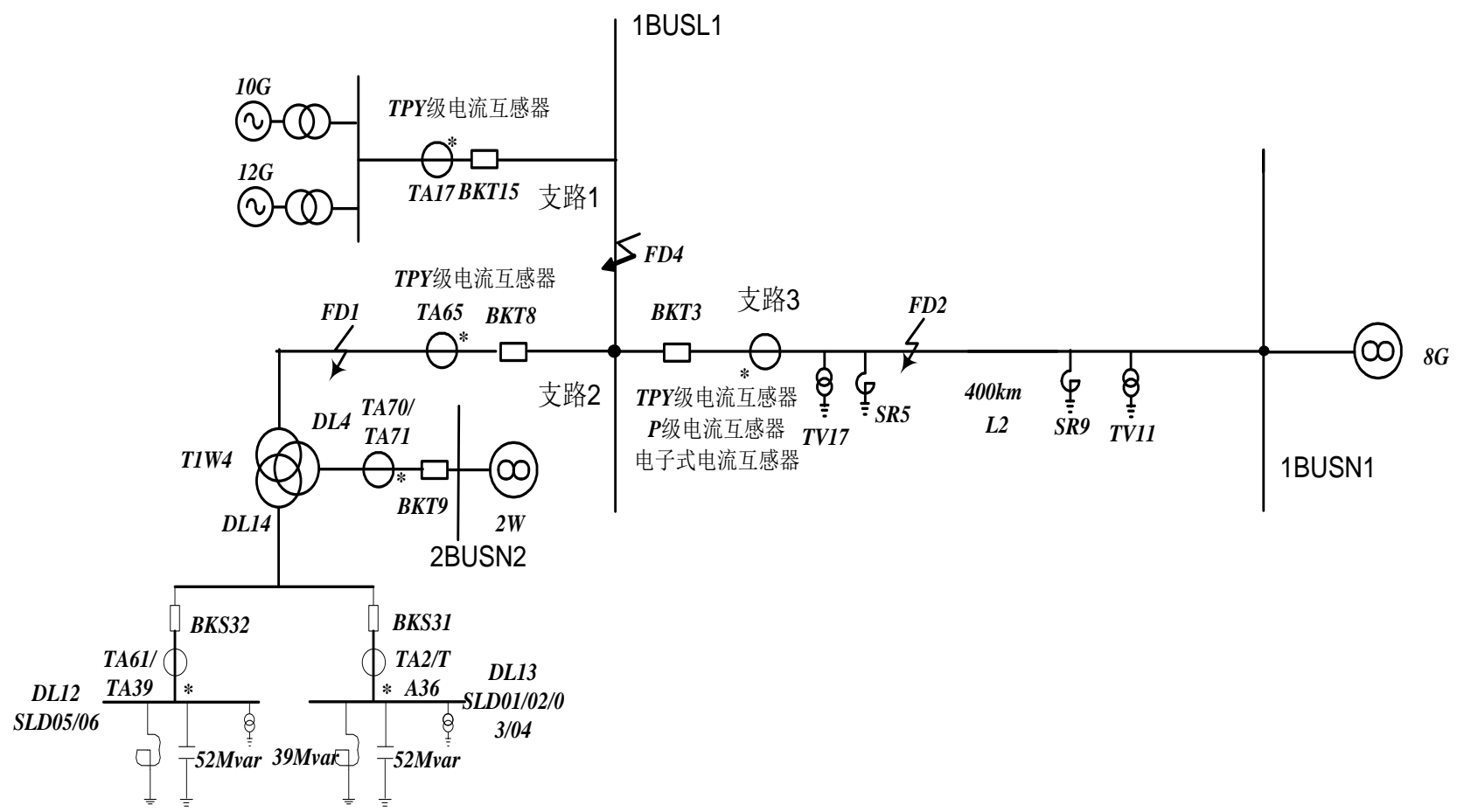

Figure 3. Dynamic model testing system. 


$$
\text { Iop }_{k}=\left\{\begin{array}{l}
0.24, \quad \text { Ires }_{k}<0.3 \\
0.6 \text { Ires }_{k}, \text { Ires }_{k} \geq 0.3
\end{array}\right.
$$

Of which, differential current $\operatorname{Iop}_{k}=\left|\dot{I}_{1}+\dot{I}_{2}+\dot{I}_{3}\right| ;$ braking current

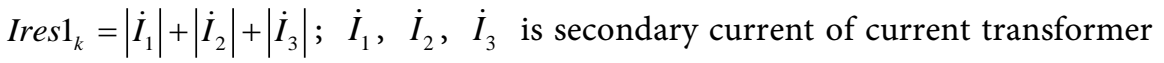
from each branch. We define the primary current flowing from bus to the outflow as the positive direction. Currently, the logic to identify current transformer saturation of the bus differential protection mostly using the time difference method, of the way to identify the current transformer saturation is based on severity of saturation, in accordance with the regulations, when the saturation time is $5 \mathrm{~ms}$ or more during an external fault, the logic criterion of the existing difference protection can judge correctly and block the differential protection, when the saturation time is less than $5 \mathrm{~ms}$ in an external fault, the differential protection has the risk of malfunction.

\subsection{Comparison Test of Multi-Type Current Transformer Transmission Performance}

As shown in Figure 3: Branch 1 configures TA1, branch 2 configures TA2, and branch 3 configures TA3. Two consecutive metal faults occur in the FD2, the fault time of which is $100 \mathrm{~ms}$, the actual waveform is shown in Figure 4. $\mathrm{imal}_{\mathrm{k}}$, $\mathrm{ima}_{\mathrm{k}}, \mathrm{ima}_{\mathrm{k}}$ represent secondary current actual waveform of P-level current transformer, TPY-level current transformer and electronic current transformer, respectively. When the current transformer is seriously saturated, waveform of the differential current and braking current is shown in Figure 5. Figure 4 shows that the difference of three current transformers transmission performance is not large when the P-level current transformer is not saturated; Figure 5 shows that the saturation time is less than 5 ms when P-class current transformer is seriously saturated in an external fault, and the time of protection malfunction is within $10 \mathrm{~ms}$.

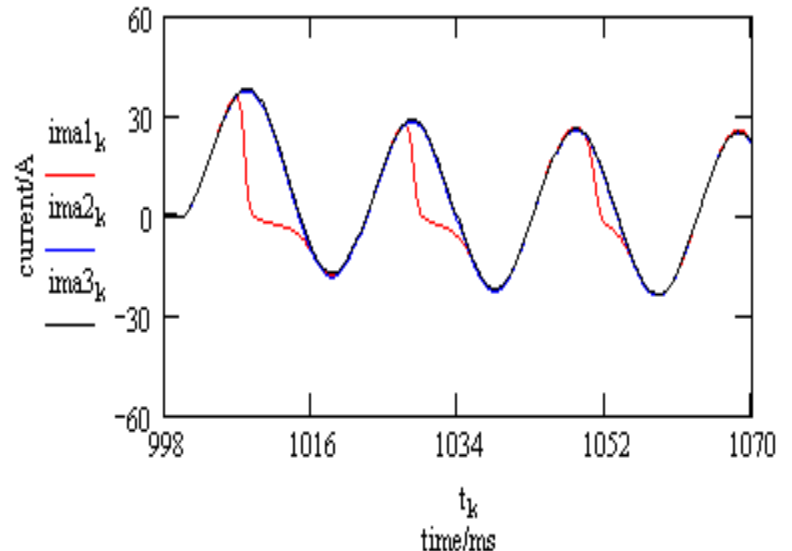

(a)

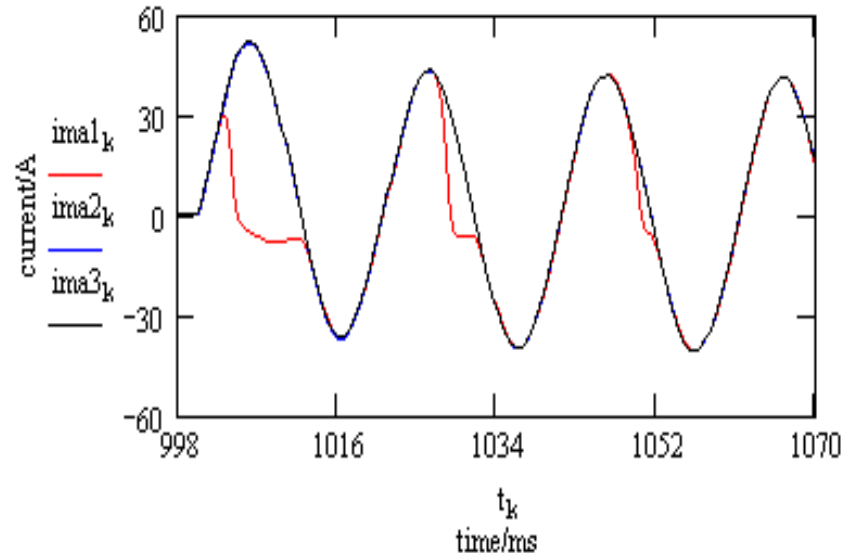

(b)

Figure 4. First external fault, external fault again secondary current actual waveform of multi type current transformer. (a) First external fault; (b) Second external fault. 


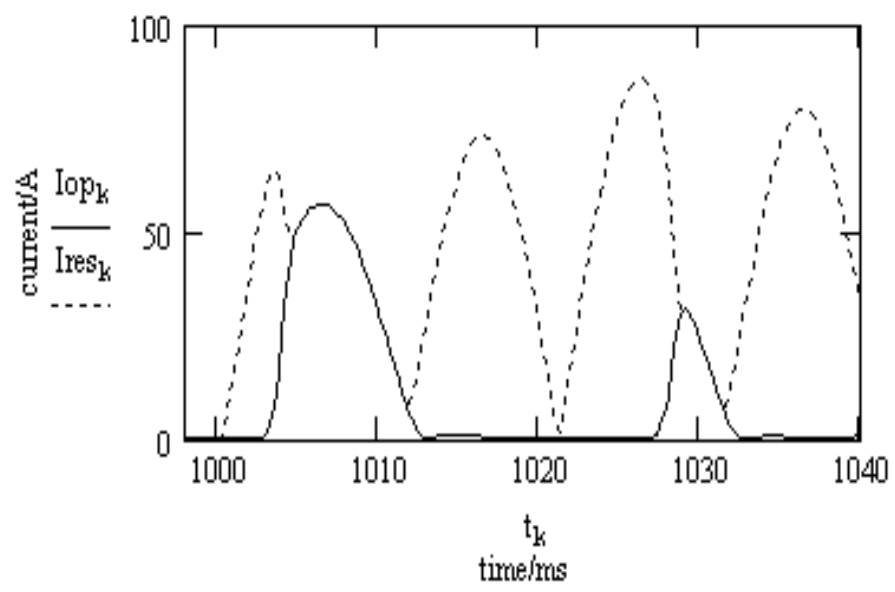

Figure 5. Waveform of differential current and braking current for current transformer serious saturation.

\subsection{Test Results Analysis of Multi-Type Current Transformers Hybrid Operation}

Branch 3 of bus is using P-level current transformer, other branches are using TPY-level current transformers. When the metal faults occur in the FD4, the track and action state are shown in Figure 6.

When the internal fault occurs in the TPY-level current transformer and electronic current transformer hybrid operation, protection device can act accurately. When P-level current transformer and TPY-level current transformer are mixedly used for bus, and if P-level current transformer is seriously saturated, protection device can still act accurately in an internal fault, although the differential current and the braking current appear at the same time and the differential current is large, which is shown in Figure 6.

The analysis of differential current after two consecutive external faults is shown in Table 1. Of which, "no differential current" means that the differential current is too small and there is no saturation time.

From Table 1, we can see that the differential current in the A and B test schemes is low and has no change when the two consecutive external faults occur, and the differential protection malfunction will not happen. Under the $\mathrm{C}$ test scheme, the saturated time of the two consecutive faults change obviously in the FD2, and the differential current is comparatively large. The track and action state are shown in Figure 7. There is no remanence in P-level current transformer at first, after the first external fault, the excitation flux gradually increases, and transient saturation occurs in about $6.5 \mathrm{~ms}$,. The current then transfers in the non-linear method, the differential current, is large and differential current appearing time is greater than $5 \mathrm{~ms}$. The track and action curves are shown in Figure 7(a). After the external re-fault, P-level current transformer has remanence. And the remanence of closed-core P-level current transformer is comparatively large, if the remanent magnetic flux and non-periodic component flux is in the same direction, the current transformer will be seriously saturated, the current will transfers in non-linear quickly, the differential current is large and 


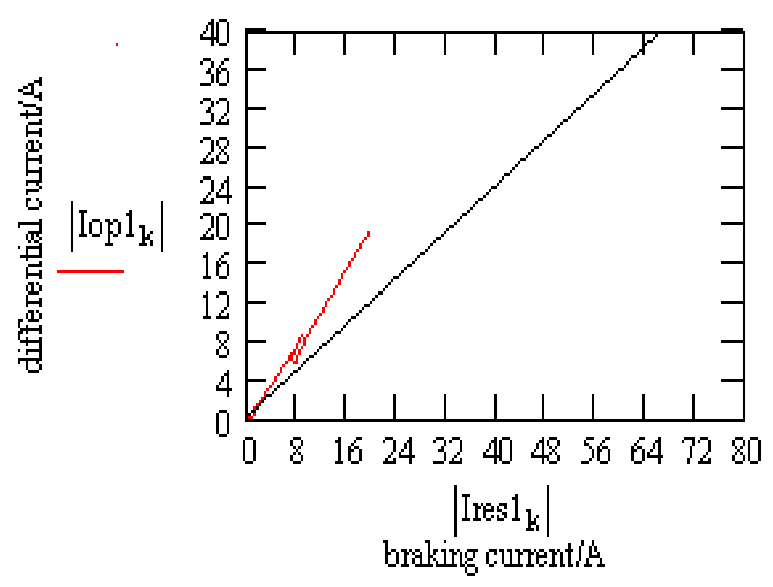

Figure 6. Internal fault track and action state for the line differential protection (Branch 3 with the P-level current transformer, and other branches with TPY-level current transformer).



(a)

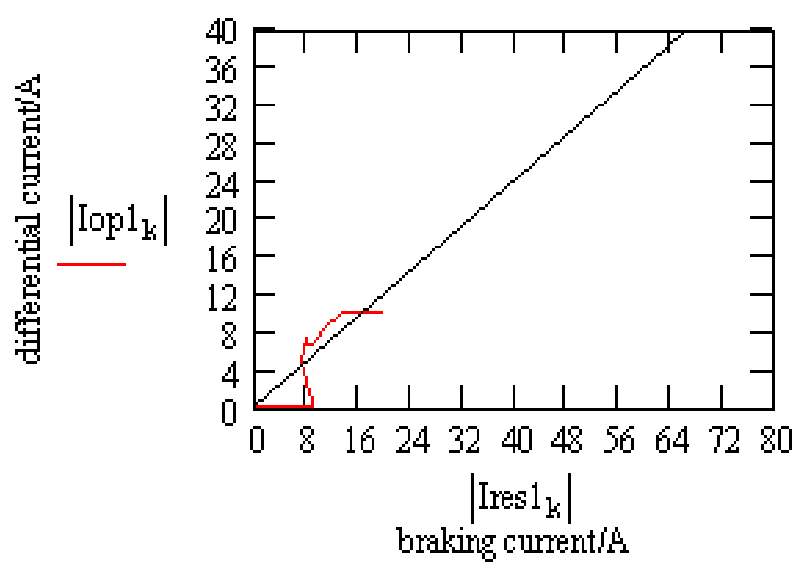

(b)

Figure 7. The first external fault, external fault again action track and action state for the line differential protection (Branch 3 with the P-level current transformer, other branches with TPY-level current transformer). (a) First external fault; (b) Second external fault. 
Table 1. Simulation results.

\begin{tabular}{|c|c|c|c|c|}
\hline \multirow{2}{*}{ Test scheme } & \multicolumn{2}{|c|}{$\begin{array}{l}\text { Appearing differential current time after } \\
\text { fault (ms) }\end{array}$} & \multicolumn{2}{|c|}{$\begin{array}{l}\text { Maximum differential current } \\
\text { (A) }\end{array}$} \\
\hline & The first fault & Re-fault & The first fault & Re-fault \\
\hline A & $\begin{array}{l}\text { No differential } \\
\text { current }\end{array}$ & $\begin{array}{l}\text { No differential } \\
\text { current }\end{array}$ & 0.11 & 0.11 \\
\hline B & $\begin{array}{l}\text { No differential } \\
\text { current }\end{array}$ & $\begin{array}{l}\text { No differential } \\
\text { current }\end{array}$ & 0.26 & 0.28 \\
\hline C & 6.5 & 2.6 & 15.11 & 15.79 \\
\hline
\end{tabular}

current appearing time is less than $5 \mathrm{~ms}$. The track and action curves are as shown in Figure 7(b).

From the above test results analysis, we can see that when the internal fault occurs, protection device can act accurately based on the transmission characteristics of the different types current transformer. While under the $\mathrm{C}$ test scheme, when the first external fault occurs, P-level current transformer enters the saturated state after $6.5 \mathrm{~ms}$, greater than the normal transmission time of the current transformer $5 \mathrm{~ms}$, so the existing differential protection device based on $5 \mathrm{~ms} \mathrm{sa}-$ turation logic criteria may correctly determine and block the differential protection and differential protection malfunction does not happen. When the external fault occurs again, the electromagnetic current transformer quickly enters the saturated state after about $2.5 \mathrm{~ms}$, less than the normal transmission time of the current transformer $5 \mathrm{~ms}$, so the time difference method based on the saturated criterion may fail. As can be seen from Figure 5, the time window for protection against malfunction may be close to $10 \mathrm{~ms}$ in the worst case of saturation. At the same time, we can see from Figure 4 that the secondary current non-periodic components of the current transformer will be significantly reduced if the electromagnetic current transformer saturation is more serious. Therefore, in the case of the current transformers hybrid operation, the normal transmission time of P-level current transformers is less than $5 \mathrm{~ms}$ and the current transformer saturation cannot be determined. If the non-periodic component change is greater than a certain value, protection based on exports fixed delay $10 \mathrm{~ms}$ can avoid the serious saturation effect of the current transformer.

\section{Conclusion}

This paper studied the effect of the current transformer hybrid operation on bus differential protection based on the dynamic simulation experiment platform. Through the analysis of the current transformer non-hybrid operation and hybrid operation and the contrast of the action characteristics of the differential protection in the internal and external fault, we can obtain the following conclusion: when the internal fault occurs, protection device of the three current transformer hybrid operation can act accurately. As the differential current of each branch of the bus for the TPY-level current transformer and electronic current transformer is very small in the external fault, the differential protection 
is not affected. When the TPY-level current transformer and P-level current transformer is used in the bus, when the first external fault occurs, P-level current transformer appears a slight saturation, the differential current is large, the time that the differential current appears is more than $5 \mathrm{~ms}$. When the external re-fault occurs, P-level current transformer is seriously saturated, the differential current is larger, the time that the differential current appears is less than $5 \mathrm{~ms}$, and the differential protection has the risk of malfunction.

\section{References}

[1] Yuan, J.X., Sheng, H.L. and Wu, J.Y. (2004) Protection of Current Transformer Application Guide. China Electric Power Press, Beijing.

[2] DL/T866-2004 (2004) Selection and Calculation of Current Transformer and Voltage Transformer.

[3] Hou, H., You, D.H., Yin, X.G., Chen, W. and Xu, T.Q. (2006) Influence and Application of Electronic Current Transducer on Distance Protection. Power System Technology, 30, 349-353.

[4] Ji, H.Q., Zhang, J., Yang, Y.H., Li, Y.S. and Guo, Z.Z. (2006) Comment on Performance of Differential Protection Taking into Account Electronic Current Transducers. Power System Technology, 30, 61-65.

[5] Luo, S.N., Tian, Z.B. and Zhao, X.C. (2004) Performance Analysis of Air-Core Current Transformer. Proceedings of the CSEE, 24, 108-113.

[6] Zhou, Z.X., Zhou, C.X., Dong, M.H., Du, D.X. and Zhang, X.L. (2008) Construction of Dynamic Simulation Lab in SGCC Simulation Center and Research of Protective Relay Test. Power System Technology, 32, 50-55.

[7] Zhou, Z.H., Zhou, C.X., Zhang X.L., et al. (2007) Experimental Study on 1000kV AC System Dynamic Simulation and Relay Protection. China Electric Power Research Institute, Beijing.

\section{Submit or recommend next manuscript to SCIRP and we will provide best service for you:}

Accepting pre-submission inquiries through Email, Facebook, LinkedIn, Twitter, etc. A wide selection of journals (inclusive of 9 subjects, more than 200 journals)

Providing 24-hour high-quality service

User-friendly online submission system

Fair and swift peer-review system

Efficient typesetting and proofreading procedure

Display of the result of downloads and visits, as well as the number of cited articles

Maximum dissemination of your research work

Submit your manuscript at: http://papersubmission.scirp.org/

Or contact epe@scirp.org 